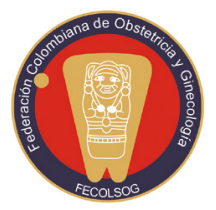

Revista Colombiana de Obstetricia y Ginecología Vol. 69 No. 2 • Abril-Junio 2018 • (88-97)

\title{
FRECUENCIA DE LA REALIZACIÓN DE EPISIOTOMÍA Y COMPLICACIONES EN EL SERVICIO DE OBSTETRICIA DEL HOSPITAL UNIVERSITARIO SAN JOSÉ, POPAYÁN (COLOMBIA), 2016. EXPLORACIÓN DE FACTORES MATERNOS Y PERINATALES ASOCIADOS A SU REALIZACIÓN
}

\section{Frequency of episiotomy and complications in the Obstetrics Service of Hospital Uliniversitario San José, Popayán (Colombia), 2016. Exploration of maternall and perinatal factors associated with its} performance

\author{
Andrés Martín Mellizo-Gaviria, MD' ${ }^{1}$; Lina María López-Veloza, $\mathbf{M D}^{2}$; \\ Richard Montoya-Mora, $\mathrm{MD}^{2}$; Roberth Alirio Ortiz-Martínez, MD, $\mathrm{MSc}^{3}$; \\ Claudia Consuelo Gil-Walteros, $\mathrm{MD}^{4}$ \\ Recibido: junio 27/17 - Aceptado: mayo 28/18
}

\section{RESUMEN}

Objetivo: determinar la frecuencia de la realización de la episiotomía, explorar los factores asociados a esta, y describir resultados maternos y perinatales en el servicio de obstetricia del Hospital Universitario San José de Popayán (Colombia) en el primer semestre del año 2016.

Materiales y métodos: estudio descriptivo de corte transversal, con análisis secundario; se incluyeron

1 Médico interno, Facultad de Medicina, Universidad del Cauca, Popayán (Colombia).andresmellizo@unicauca.edu.co

2 Médico interno, Facultad de Medicina, Universidad del Cauca, Popayán (Colombia).

3 Especialista en Ginecología y Obstetricia; magíster en Epidemiología. Docente del Departamento de Ginecología y Obstetricia, Universidad del Cauca, Popayán (Colombia).

4 Especialista en Ginecología y Obstetricia. Docente del Departamento de Ginecología y Obstetricia, Universidad del Cauca, Popayán (Colombia). gestantes con embarazo mayor de 37 semanas cuyos partos fueron atendidos el primer semestre del año 2016, en un hospital público de alta complejidad, centro de referencia del departamento del Cauca (Colombia), el cual atiende población del aseguramiento contributivo y subsidiado. Se realizó un muestreo aleatorio simple, con tamaño de muestra de 197 partos, y margen de error del $5 \%$. Se evaluaron variables maternas, del parto, de resultado materno y neonatal. Se estimó la frecuencia de episiotomía y se realizó exploración de los factores asociados a esta por medio de análisis bivariado y multivariado.

Resultados: la frecuencia de la realización de episiotomía fue de 30,45\% (n=60; IC $95 \%$ : 24,1-37,3), la complicación más frecuente fue el desgarro perineal, con $29 \%$ (IC95\%: 22,9-35,5). 
En cuanto a los factores de riesgo, la nuliparidad fue el único factor asociado al uso de la episiotomía $($ Ora $=16,11$; IC $95 \%:$ 6,46-42,81).

Conclusión: el uso de la episiotomía en esta institución es superior a lo recomendado por la Organización Mundial de la Salud (OMS). Se deben evaluar estrategias para reducir su frecuencia a los niveles esperados.

Palabras clave: episiotomía, paridad, perineo, parto, obstétrico.

\section{ABSTRACT}

Objective: To determine the frequency with which episiotomy is performed, explore factors associated with its performance, and describe maternal and perinatal outcomes in the obstetric service of San José University Hospital in the city of Popayán (Colombia) during the first semester of 2016.

Materials and methods: Descriptive, crosssectional study with secondary analysis which included pregnant women with more than 37 weeks of gestation delivered during the first semester of 2016 in a high complexity public referral centre in the Department of Cauca Colombia, which serves patients covered by both the contributive as well as the subsidised health insurance regimes. A simple random sampling was used with a sample size of 197 deliveries and a margin of error of 5\%. Maternal and childbirth variables, as well as maternal and neonatal outcomes were assessed. The frequency of episiotomy was estimated and the factors associated with its performance were explored by means of bivariate and multivariate analysis.

Results: The frequency with which episiotomy was performed was 30.45\% ( $\mathrm{n}=60$; 95\% CI: 24.1-37.3), and the most frequent complication was perineal tear at 29\% (95\% CI: 22.9-35.5). In terms of risk factors, nulliparity was the only factor associated with the need to perform episiotomy $(\mathrm{OR}=16.11$; 95\% CI: 6.46-42.81).

Conclusion: Episiotomy is performed more frequently in this institution than recommended by the World Health Organisation (WHO). Strategies should be considered for reducing this frequency to the expected levels.

Key words: Episiotomy, parity, perineum, delivery, obstetric.

\section{INTRODUCCIÓN}

La episiotomía es un procedimiento quirúrgico realizado para ampliar la parte inferior de la vagina, el anillo vulvar y el tejido perineal durante la fase de expulsión fetal en el parto (1). Es uno de los procedimientos más comunes en obstetricia, a pesar de que la evidencia científica actual no apoya su práctica rutinaria (2-4). Existen cuatro técnicas para realizar una episiotomía: línea media, mediolateral, lateral y en forma de "J" (5). Se ha usado históricamente para evitar desgarros vaginales espontáneos (6,7), disminuir la mortalidad y morbilidad del recién nacido $(2,8,9)$. Además, se ha argumentado que una incisión quirúrgica controlada es generalmente más fácil de reparar (6).

Las tendencias internacionales actuales son hacia la disminución de la realización de episiotomía y a limitar su uso a las indicaciones específicas (10). El uso restrictivo de este procedimiento en partos vaginales sin complicaciones, en oposición a la episiotomía de rutina, se ha asociado a un menor riesgo de traumatismo perineal posterior y necesidad de sutura $(11,12)$. Su realización varía de un país a otro, encontrándose cifras reportadas desde el 8 al $95 \%$, las cuales varían en relación con la frecuencia de parto instrumentado, parto pretérmino, parto en pelvis, sospecha de macrosomía fetal o desgarro perineal inminente $(5,11-14)$. Al no haber evidencia confiable para el uso rutinario, o efectos benéficos, la Organización Mundial de la Salud (OMS) recomienda que la frecuencia de su uso no sea mayor al $10 \%(13)$.

Es escasa la información sobre la frecuencia de la realización de este procedimiento y los factores asociados, a nivel nacional (4) y regional $(15,16)$. Es importante a nivel institucional conocerla para 
poder definir si se está realizando dentro de los estándares sugeridos internacionalmente a fin de planear acciones de mejoramiento de calidad en los servicios de obstetricia que lleven a un uso racional del procedimiento. De esta manera, la presente investigación tiene como objetivo principal determinar la frecuencia de la realización y las complicaciones asociadas y, secundariamente, explorar los factores asociados a la episiotomía y describir los resultados maternos y neonatales en el servicio de obstetricia del Hospital Universitario San José (HUSJ) de Popayán (Colombia), en el primer semestre del año 2016.

\section{MATERIALES Y MÉTODOS}

Diseño y población. Se desarrolló Se desarrolló un estudio descriptivo de corte transversal, en gestantes con parto vaginal, con edad gestacional mayor o igual a 37 semanas, atendidas en el HUSJ de la ciudad de Popayán, en el primer semestre del año 2016. Esta institución general presta servicios de tercer nivel de complejidad, y es centro de referencia en el departamento del Cauca, en la región suroccidental del país, del país, y atiende población perteneciente al régimen contributivo y al subsidiado por el Estado en el sistema de seguridad social en Colombia. Se excluyeron pacientes con historia clínica y evolución incompleta o con pérdida de datos mayor al 10\%. Se estimó un tamaño de muestra de 197 pacientes, con base en la fórmula: $\mathrm{n}=\mathrm{P} \times \mathrm{Q} /(\mathrm{E} / \mathrm{Z})^{2}$, se tuvo en cuenta la población promedio de nacidos vivos en los años previos en la institución (1800 partos, 900 cesáreas y 900 partos vaginales en el 2015), con una frecuencia esperada de episiotomía del 20\% (cercana a la propuesta por la OMS, del $10 \%$ ) (13), el margen de error tolerado fue del $5 \%$, con un nivel de confianza del $95 \%$.

Procedimiento. Se identificaron las pacientes gestantes con parto vaginal, con edad gestacional mayor o igual a 37 semanas, dadas por ecografía temprana o fecha de última menstruación confiable, y se realizó un muestreo aleatorio simple por medio de un listado de números aleatorios generado en el programa Microsoft Excel; previo a la recolección de la información en el posparto se solicitó la firma del consentimiento informado a las gestantes por parte de los investigadores. Luego se diligenció el formulario a partir de la historia clínica institucional. Se creó una base de datos en Excel utilizando reglas de validación para el control de las entradas a fin de garantizar la confiabilidad y calidad de los datos, el análisis se realizó utilizando el software Stata versión 9 con posterior encriptación de los datos.

Variables por medir. Se midieron las siguientes variables: características de la gestante: edad materna, procedencia, peso $(\mathrm{kg})$, talla $(\mathrm{cm})$ (para el cálculo del índice de masa corporal - IMC en kg/ $\mathrm{m}^{2}$ ), paridad, controles prenatales adecuados (cuatro o más controles prenatales iniciados durante el primer trimestre), edad gestacional, patología materna obstétrica (trastornos hipertensivos del embarazo, ruptura prematura de membranas, corioamnionitis, diabetes gestacional). Características del parto: duración del expulsivo y del trabajo de parto en minutos, inducción del trabajo de parto, parto instrumentado, presentación, retención de hombros, peso del recién nacido. Variables de resultado - Maternos: episiotomía, presencia de desgarro, clasificación del desgarro en grados I-IV (17), infección puerperal y hemorragia posparto. Neonatales: APGAR al minuto, a los 5 minutos y a los 10 minutos; líquido amniótico meconiado, requerimiento de unidad de cuidados intensivos neonatales (UCIN) o cuidados intermedios, y si presentó o no síndrome de dificultad respiratoria aguda (SDRA).

Análisis estadístico. Para determinar la frecuencia se tomó en cuenta como numerador el total de pacientes a quienes se les había realizado episiotomía, y como denominador el total de pacientes con parto vaginal que cumplieron los criterios de inclusión. Las variables sociodemográficas y clínicas basales se presentan comparando el grupo que recibió episiotomía con el que no la recibió. Las variables 
continuas se resumen por medio de medidas de tendencia central y dispersión, las categóricas como proporciones. En las variables continuas se evaluó la normalidad por medio del test de normalidad de Shapiro Wilk. Las variables de distribución normal se compararon por medio de la prueba $t$ de Student, y las de distribución no normal por medio de la prueba Mann-Whitney; las variables categóricas se compararon por la prueba chi cuadrado o test de Fisher. Se realizó un análisis bivariado con las variables establecidas. Se estableció la asociación por medio de la razón de prevalencia con su respectivo intervalo de confianza del 95\% (IC 95\%). Finalmente, se realizó un análisis multivariado por regresión logística para establecer la asociación entre episiotomía y primiparidad, ajustando por los posibles factores de confusión. Se empleó el procedimiento stepwise con una probabilidad de entrada de 0,20 y salida 0,05; además del criterio estadístico para la selección de variables se consideró el criterio clínico. El análisis de la información obtenida se realizó mediante el programa estadístico Stata versión 9.

Aspectos éticos. El estudio y el análisis del mismo se realizaron con la base de datos del HUSJ, avalado mediante acta de aprobación 10 del 21 de noviembre de 2015. Se solicitó la firma del consentimiento informado a las mujeres que participaron y se garantizó la confidencialidad de la información.

\section{RESULTADOS}

En el intervalo de tiempo comprendido entre enero-junio de 2016, se atendieron en la institución 448 pacientes con parto vaginal, de los cuales 323 cumplieron los criterios de inclusión, 125 (39\%) se excluyeron por pérdida de datos mayor al $10 \%$ y evolución incompleta. Se obtuvo una muestra aleatoria simple de 197 pacientes (figura 1). Se realizó episiotomía en 60 gestantes con una frecuencia de episiotomía del 30,45\% (IC $95 \%$ : 24,1-37,3).

$\mathrm{Al}$ comparar los grupos de pacientes con y sin episiotomía con respecto a las características so- ciodemográficas y clínicas basales se encontraron diferencias clínica y estadísticamente significativas en la edad materna y la paridad. Aunque el IMC mostró diferencias estadísticas, estas no fueron clínicamente relevantes. No hubo diferencias en la patología materna previa al tiempo del expulsivo o en el peso del recién nacido (tabla 1). En el análisis bivariado de los factores asociados a uso de episiotomía se encontró que las pacientes sin partos previos $(\mathrm{RP}=8,81$; IC $95 \%$ : 4,22-18,4) y menores a 19 años $(\mathrm{RP}=1,76$; IC $95 \%: 1,14-2,63)$ presentaron mayor frecuencia de realización de episiotomía por parte del médico tratante. Fueron factores protectores el IMC > $25(\mathrm{RP}=0,51 ;$ IC $95 \%: 0,34-0,77)$ y la edad mayor a 34 años (RP = 0,12; IC $95 \%$ : 0,01$0,83)$, no hubo diferencias respecto a si el parto fue inducido o espontáneo, parto instrumentado o feto con peso mayor a $3999 \mathrm{~g}$ (tabla 2).

Respecto a los resultados maternos y perinatales se encontró que las pacientes con episiotomía presentaron menor frecuencia de desgarros: 3 $(5,26 \%)$ frente a $54(39,42 \%)$ de las pacientes sin episiotomía(RP= 0,12; IC95\%: 0,04-0,39). El $28,93 \%(n=57)$ de los partos atendidos presentaron desgarro perineal, siendo grado I el 16,75\% $(n=33)$, grado II el 11,68\% ( $=23)$, grado III el 0,51\% (n= 1), y ninguno grado IV. No se presentaron infecciones o hemorragias posparto. Para los resultados neonatales no se encontraron diferencias en cuanto a la presencia de líquido amniótico meconiado $(\mathrm{RP}=0,68$; IC $95 \%$ : 0,19-2,4), ingreso a UCIN $(\mathrm{RP}=1,59$; IC $95 \%$ : 0,63-3,99) o síndrome de dificultad respiratoria (tabla 3 ).

El análisis multivariado se ajustó por variables que se consideraron clínicamente importantes desde el punto de vista de la obstetricia: peso fetal, edad materna, paridad, IMC; se encontró significancia estadística únicamente en la paridad (ORa $=16,11$; IC 95 \%: 6,17-42,81) (tabla 4). No se pudo realizar análisis con la duración del expulsivo ya que no se presentaron expulsivos prolongados. 
Figura 1.

Flujograma de pacientes

448 partos vaginales

Primer semestre 2016

Cumplieron criterios de inclusión: 323 partos vaginales Se excluyeron: 125 por pérdida de datos mayor al $10 \%$
Muestreo aleatorio simple: 197 partos vaginales

Población definitiva

de estudio: 197 partos

vaginales

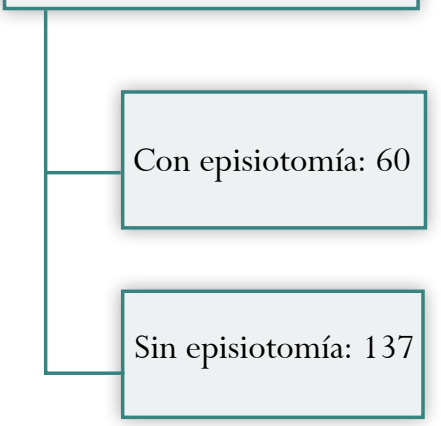

\section{DISCUSIÓN}

Durante los meses de enero a junio del 2016, se obtuvo una frecuencia de episiotomías realizadas del 30,45\%, siendo la nuliparidad el principal factor asociado; con la realización de este procedimiento se presentó menor frecuencia de desgarros.

Respecto a la frecuencia de episiotomía nuestros resultados se asemejan a lo reportado en estudios como el realizado por Pérez Valero et al., que reportan una frecuencia del 33,5\% (18), así como a otros estudios que muestran una frecuencia de 29,9 y 29,1\%, realizados en poblaciones similares y con políticas restrictivas parecidas (15-18); sin embargo, otros estudios latinoamericanos realizados en po- blaciones similares reportaron entre el 49 y el $61 \%$, entre los años 2005 y 2009, momentos en que se empiezan a implementar políticas restrictivas para mejorar la atención materna (19-21).

Similar a lo encontrado en nuestro estudio, Campos Braga et al. encontraron la primiparidad como factor de riesgo $(\mathrm{OR}=3,08$; IC $95 \%$ : 2,16-4,41) (20), resultados reportados también por el estudio de Trinh et al. (OR = 2,22; IC $95 \%: 1,48-3,32)$ (17). No hubo asociación con la patología materna previa, similar al estudio de Trinh et al., donde estudian los trastornos hipertensivos del embarazo $(\mathrm{OR}=1,04$; IC $95 \%$ : 0,77-1,36) y diabetes mellitus $(\mathrm{OR}=1,04$; IC 95 \%: 0,77-1,36) (17). En cuanto a la inducción 


\begin{tabular}{|c|c|c|c|}
\hline Variable & Episiotomía $(+) n=60$ & Episiotomía (-) n = 137 & Valor $\mathbf{p}$ \\
\hline Edad* & $22,3( \pm 5,7)$ & $27,7( \pm 7,3 \mathrm{DS})$ & 0,000 \\
\hline IMC & $26,6( \pm 3,3)$ & $28( \pm 3,7)$ & 0,010 \\
\hline Paridad $\uparrow$ & $0(0)$ & $1(2)$ & 0,000 \\
\hline CPN adecuado ${ }^{\ddagger}$ & $42(32,5 \%)$ & $87(67,4 \%)$ & 0,377 \\
\hline Edad gestacional (en días) & $274,3( \pm 6,7)$ & $275,3( \pm 7,5)$ & 0,354 \\
\hline Hipertensión & $7(29,17 \%)$ & $17(70,83 \%)$ & 0,372 \\
\hline $\mathrm{RPM}^{\ddagger}$ & $4(44,44 \%)$ & $5(55,56 \%)$ & 0,372 \\
\hline Corioamnionitis ${ }^{\ddagger}$ & $0(0 \%)$ & $1(100 \%)$ & 0,372 \\
\hline Diabetes gestacional ${ }^{*}$ & $0(0 \%)$ & $1(100 \%)$ & 0,372 \\
\hline Duración del trabajo de parto ${ }^{\dagger}$ & $6(5,5)$ & $6(3)$ & 0,326 \\
\hline Duración del expulsivo $^{\dagger}$ & $60(30)$ & $30(30)$ & 0,296 \\
\hline Inducción ${ }^{\ddagger}$ & $15(28,85 \%)$ & $37(71,15 \%)$ & 0,769 \\
\hline Parto instrumentado & $1(25 \%)$ & $3(75 \%)$ & 0,811 \\
\hline Peso del recién nacido* & $3193,6( \pm 373,2)$ & $3243,8( \pm 355)$ & 0,369 \\
\hline $\begin{array}{l}\text { Fuente: datos del estudio. } \\
* \text { t Student } \\
\dagger \text { prueba de rangos } \\
\text { † Chi cuadrado }\end{array}$ & & & \\
\hline
\end{tabular}

del parto, nuestro estudio no encontró asociación con la realización de episiotomías, en contraposición a lo descrito en otros estudios como el realizado por Campos Braga et al., donde se reportó como factor de riesgo $(\mathrm{OR}=1,92$; IC $95 \%$ : 1,31-2,79). No se encontró asociación con el parto instrumentado, en contraste con el anterior estudio, el cual reporta mayor riesgo en pacientes a quienes se les realiza $(\mathrm{OR}=18,91 ;$ IC $95 \%:$ 7,86-45,48). Además, en el mismo estudio no hubo asociación con el peso al nacer > $3999 \mathrm{~g}(\mathrm{OR}=1,12$; IC $95 \%$ : 0,37-3,41) similar a lo encontrado en nuestra población (20).

Al igual que en nuestro estudio, Santos Oliveira et al. reportaron mayor frecuencia de desgarros sin la realización de episiotomía $(\mathrm{OR}=26,03$; IC $95 \%$ : 18,13-37,37) (21).
El presente estudio tiene como fortalezas la muestra representativa, la variedad de factores asociados analizados y el manejo de factores confusores por medio del análisis multivariado. Como limitaciones, el número de sujetos excluidos por información incompleta (39\%), el hecho de que se realizó en una sola institución de tercer nivel y esta no puede extrapolarse a toda la región; y que además no se evaluaron las prácticas, los conocimientos y la actitud del obstetra, variables que pueden incidir en la decisión de la episiotomía.

\section{CONCLUSIÓN}

La frecuencia de episiotomía encontrada en el año 2016 en el HUSJ de Popayán fue del 30,45\%. Las pacientes sin partos previos presentaron 
Tabla 2.

Análisis bivariado que evalúa los factores asociados al uso de la episiotomía en el Hospital Universitario San José de Popayán (Colombia), 2016

\begin{tabular}{|c|c|c|c|c|}
\hline \multirow[b]{2}{*}{ Factores asociados } & \multicolumn{2}{|c|}{ Episiotomía } & \multirow[b]{2}{*}{$\mathbf{R P}$} & \multirow[b]{2}{*}{ IC $95 \%$} \\
\hline & $\begin{array}{l}\text { Sí } \\
\mathrm{n}=60(30,46 \%)\end{array}$ & $\begin{array}{c}\text { No } \\
\mathrm{n}=137(69,54 \%)\end{array}$ & & \\
\hline \multicolumn{5}{|l|}{ Edad (años) } \\
\hline$<19$ & $16(53,33)$ & $14(46,67)$ & 1,76 & $1,14-2,63$ \\
\hline $19-34$ & $43(30,71)$ & $97(59,29)$ & REF & - \\
\hline$>34$ & $1(3,7)$ & $26(96,3)$ & 0,12 & $0,01-0,83$ \\
\hline \multicolumn{5}{|l|}{ Procedencia } \\
\hline Urbano & $42(31,34)$ & $92(68,66)$ & REF & - \\
\hline Rural & $18(28,57)$ & $45(71,43)$ & 0,91 & $0,57-1,44$ \\
\hline \multicolumn{5}{|l|}{ IMC } \\
\hline Adecuado $(18-24,9)$ & $25(47,17)$ & $28(52,83)$ & REF & - \\
\hline Sobrepeso y obesidad $(>25)$ & $35(24,3)$ & $109(75,69)$ & 0,51 & $0,34-0,77$ \\
\hline \multicolumn{5}{|l|}{ Paridad } \\
\hline Primiparidad o multiparidad & $7(6,6)$ & $99(93,4)$ & REF & - \\
\hline Nuliparidad & $53(58,24)$ & $38(41,76)$ & 8,81 & $4,22-18.4$ \\
\hline \multicolumn{5}{|l|}{ Control prenatal } \\
\hline Adecuado & $42(32,56)$ & $87(67,44)$ & REF & - \\
\hline No adecuado & $18(26,47)$ & $50(73,53)$ & 0,81 & $0,5-1,29$ \\
\hline \multicolumn{5}{|l|}{ Patología obstétrica } \\
\hline No & $48(29,63)$ & $114(70,37)$ & REF & - \\
\hline Sí & $12(34,29)$ & $23(65,71)$ & 1,15 & $0,69-1,93$ \\
\hline \multicolumn{5}{|c|}{ Inducción del trabajo de parto } \\
\hline No & $45(31,03)$ & $100(68,97)$ & REF & - \\
\hline Sí & $15(28,85)$ & $37(71,15)$ & 0,92 & $0,56-1,51$ \\
\hline \multicolumn{5}{|l|}{ Parto instrumentado } \\
\hline No & $59(30,57)$ & $134(69,43)$ & REF & - \\
\hline Sí & $1(25)$ & $3(75)$ & 0,81 & $0,14-4,52$ \\
\hline \multicolumn{5}{|l|}{ Peso del recién nacido (g) } \\
\hline$<2500$ & $3(75)$ & $1(25)$ & 2,49 & $1,35-4,56$ \\
\hline $2500-3999$ & $56(30,11)$ & $130(69,89)$ & REF & - \\
\hline > 3999 & $1(14,29)$ & $6(85,71)$ & 0,47 & $0,07-2,95$ \\
\hline
\end{tabular}

Fuente: datos del estudio.

RP: Razón de prevalencia; IC: Intervalo de confianza; REF: Referencia; IMC: Índice de masa corporal 


\begin{tabular}{|c|c|c|c|c|c|c|c|c|}
\hline \multirow{3}{*}{ Episiotomía } & \multicolumn{8}{|c|}{ Resultados maternos y perinatales } \\
\hline & \multicolumn{2}{|c|}{ Desgarro } & \multicolumn{2}{|c|}{ LAM } & \multicolumn{2}{|c|}{ UCIN } & \multicolumn{2}{|c|}{ SDRA } \\
\hline & Sí & No & Sí & No & Sí & No & Sí & No \\
\hline Sí & $3(5 \%)$ & $57(95 \%)$ & $3(5 \%)$ & $57(95 \%)$ & $\begin{array}{c}7 \\
(11,66 \%)\end{array}$ & $\begin{array}{c}53 \\
(88,33 \%)\end{array}$ & $0(0 \%)$ & $60(100 \%)$ \\
\hline No & $\begin{array}{c}54 \\
(39,41 \%)\end{array}$ & $\begin{array}{c}83 \\
(60,58 \%)\end{array}$ & $\begin{array}{c}10 \\
(7,29 \%)\end{array}$ & $\begin{array}{c}127 \\
(92,7 \%)\end{array}$ & $\begin{array}{c}23 \\
(16,78 \%)\end{array}$ & $\begin{array}{c}114 \\
(83,21 \%)\end{array}$ & $3(2,18 \%)$ & $\begin{array}{c}134 \\
(97,81 \%)\end{array}$ \\
\hline $\mathrm{RP}$ & 0,12 & REF & 0,68 & REF & 1,59 & REF & 0 & REF \\
\hline IC $95 \%$ & $0,04-0,39$ & - & $0,19-2,4$ & - & $\begin{array}{c}0,63- \\
3,99\end{array}$ & - & - & - \\
\hline
\end{tabular}

Fuente: datos del estudio.

RP: Razón de prevalencia; IC: Intervalo de confianza; REF: Referencia; LAM: Líquido amniótico meconiado, UCIN: Unidad de cuidados intensivos neonatales; SDRA: Síndrome de dificultad respiratoria aguda

Tabla 4.

Modelo multivariado que evalúa los principales factores asociados al uso de la episiotomía en el Hospital Universitario San José de Popayán (Colombia), 2016

\begin{tabular}{|l|c|c|c|}
\hline \multicolumn{2}{|c|}{ Factores asociados } & Ora & IC 95\% \\
\hline \multirow{2}{*}{ Edad (años) } & $<19$ & 0,71 & $0,28-1,8$ \\
\hline IMC & $>34$ & 0,27 & $0,028-2,6$ \\
\hline Paridad & Sobrepeso y obesidad & 0,62 & $0,27-1,4$ \\
\hline & Nuliparidad & 16,11 & $6,17-42,81$ \\
\hline Peso del recién nacido & $<2500$ & 10,38 & $0,27-393,4$ \\
\hline
\end{tabular}

Fuente: datos del estudio.

ORa: OR ajustado; IC: Intervalo de confianza; IMC: Índice de masa corporal

mayor frecuencia de realización de episiotomía por parte del médico tratante. Se deben evaluar estrategias para reducir su frecuencia a los niveles esperados.

\section{AGRADECIMIENTOS}

Agradecemos al Hospital Universitario San José (HUSJ) que nos permitió llevar a cabo el proceso de investigación dentro de sus instalaciones; $\mathrm{Al}$ departamento de Ginecología y Obstetricia de la Universidad del Cauca, por la orientación y colaboración en el desarrollo de esta investigación.

\section{FINANCIACIÓN}

El presente estudio no cuenta con financiación ni patrocinio, se realiza sin fines de lucro. 


\section{REFERENCIAS}

1. Ballesteros Meseguer C, Carrillo García C, Meseguer de Pedro M, Canteras Jordana M, Martínez Roche M. La episiotomía y su relación con distintas variables clínicas que influyen en su realización. Rev Lat Am Enfermagem. 2016;24:1-6.

2. Silva CN, Coutada RS, Rocha A. Episiotomy: Early maternal and neonatal outcomes of selective versus routine use. Acta Obstet Ginecol Port. 2014;8(2):126-34.

3. Molina Reyes C, Huete Morales M, Sánchez Pérez JC, Ortiz Albarín M, Jiménez Barragán I, Aguilera Ruiz MA. Implantación de una política de episiotomía selectiva en el Hospital de Baza. Resultados maternofetales. Prog Obstet Ginecol. 2011;54:101-8. https:// doi.org/10.1016/j.pog.2011.01.008.

4. Rubio JA. Política selectiva de episiotomía y riesgo de desgarro perineal en un hospital universitario. Rev Colomb Obstet Ginecol. 2005;56:116-26.

5. Muhleman M, Aly I, Walters A, Topale N, Tubbs R. et al. To cut or not to cut, that is the question: A review of the anatomy, the technique, risks, and benefits of an episiotomy. Clin Anat. 2017;30:362-72. https:// doi.org/10.1002/ca.22836.

6. Steiner N, Weintraub AY, Wiznitzer A, Sergienko R, Sheiner E. Episiotomy: The final cut? Arch Gynecol Obstet. 2012;286:1369-73. https://doi.org/10.1007/ s00404-012-2460-x.

7. Dim C, Chigbu C, Obiora-Izuka C, Izuka E. Prevalence and predictors of episiotomy among women at first birth in Enugu, south-east Nigeria. Ann Med Health Sci Res. 2014;4:928-32. https://doi. org/10.4103/2141-9248.144916.

8. Oliveira D, Parente M, Calvo B, Mascarenhas T, Jorge R. A biomechanical analysis on the impact of episiotomy during childbirth. Biomech Model Mechanobiol. 2016;15:1523-34. https://doi.org/10.1007/s10237016-0781-6.

9. Chehab M, Courjon M, Eckman-Lacroix A, Ramanah R, Maillet R, Riethmuller D. Influence d'une forte diminution du recours à l'épisiotomie sur le taux global de périnée intact et peu lésionnel dans une population d'une maternité de niveau III. La Revue Sage-Femme. 2014;13:278-84. https://doi. org/10.1016/j.sagf.2014.09.004.

10. Fodstad K, Staff AC, Laine K. Effect of different episiotomy techniques on perineal pain and sexual activity 3 months after delivery. Int Urogynecol J. 2014;25:1629-37. https://doi.org/10.1007/s00192014-2401-2.

11. Jiang H, Qian X, Carroli G, Garner P. Selective versus routine use of episiotomy for vaginal birth. Cochrane Database Syst Rev. 2017; Issue 2: CD000081. https:// doi.org/10.1002/14651858.CD000081.pub3.

12. American College of Obstetricians-Gynecologists. ACOG Practice Bulletin. Practice Bulletin No. 165: Prevention and Management of Obstetric Lacerations at Vaginal Delivery. Obstet Gynecol. 2016;128:e1-e15. https://doi.org/10.1097/AOG.0000000000001523

13. Grupo de Trabajo Técnico. Organización Mundial de la Salud. Cuidados en el parto normal: una guía práctica. Rev Hosp Mat Inf Ramón Sardá. 1999;18:78-80.

14. Ministerio de Salud y Protección Social (Colombia). Norma Técnica de Atención del Parto. 2007 [Visitado 2017 May 20]. Disponible en: http://207.58.191.15:8180/xmlui/bitstream/ handle/123456789/42/Legal_34.pdf?sequence $=1$.

15. Carvalho CC, Souza ASR, Moraes Filho OB. Prevalence and factors associated with practice of episiotomy at a maternity school in Recife, Pernambuco, Brazil. Rev Assoc Med Bras. 2010;56:333-9. https://doi. org/10.1590/S0104-42302010000300020

16. Maribel EM. Frequency of use of episiotomy in the service of obstetrics of the infantile maternal hospital. Rev Med La Paz. 2009;15:27-31.

17. Trinh AT, Khambalia A, Ampt A, Morris JM, Roberts CL. Episiotomy rate in Vietnamese-born women in Australia: support for a change in obstetric practice in Viet Nam. Bull World Health Organ. 2013;91,350-6. https://doi.org/10.2471/BLT.12.114314.

18. Pérez Valero S. Episiotomía en partos vaginales eutócicos en el Hospital Universitario “La Ribera”. Nure Inv. 2013;10:1-6. 
19. Arango F, Gómez JG, Zuleta JJ. Uso de prácticas clínicas durante el embarazo, parto, puerperio y recién nacido, en hospitales públicos de ManizalesColombia, 2005. Rev Colomb Obstet Ginecol. 2005;56:271-80.

20. Braga GC, Clementino STP, Luz PFND, Scavuzzi A, Noronha Neto C, Amorim MMR. Risk factors for episiotomy: A case-control study. Rev Assoc Med Bras.
2014;60:465-72. https://doi.org/10.1590/18069282.60.05.015.

21. Oliveira LS, Brito LGO, Quintana SM, Duarte G, Marcolin AC. Perineal trauma after vaginal delivery in healthy pregnant women. São Paulo Med J. 2014;132:231-8. https://doi.org/10.1590/15163180.2014 .1324710 .

\section{Conflicto de intereses: ninguno declarado.}

